\title{
Closed-form expression for BER of CE-OFDM in optical intensity-modulated direct-detection systems
}

\section{Citation for published version (APA):}

Oliari, V., Santos, C., Marciano, P., Rocha, H. R. O., Segatto, M. E. V., \& Silva, J. A. L. (2019). Closed-form expression for BER of CE-OFDM in optical intensity-modulated direct-detection systems. IEEE Communications Letters, 23(10), 1796-1800. [8765594]. https://doi.org/10.1109/LCOMM.2019.2929515

\section{Document license:}

TAVERNE

DOI:

10.1109/LCOMM.2019.2929515

Document status and date:

Published: 01/10/2019

\section{Document Version:}

Publisher's PDF, also known as Version of Record (includes final page, issue and volume numbers)

\section{Please check the document version of this publication:}

- A submitted manuscript is the version of the article upon submission and before peer-review. There can be important differences between the submitted version and the official published version of record. People interested in the research are advised to contact the author for the final version of the publication, or visit the $\mathrm{DOI}$ to the publisher's website.

- The final author version and the galley proof are versions of the publication after peer review.

- The final published version features the final layout of the paper including the volume, issue and page numbers.

Link to publication

\section{General rights}

Copyright and moral rights for the publications made accessible in the public portal are retained by the authors and/or other copyright owners and it is a condition of accessing publications that users recognise and abide by the legal requirements associated with these rights.

- Users may download and print one copy of any publication from the public portal for the purpose of private study or research.

- You may not further distribute the material or use it for any profit-making activity or commercial gain

- You may freely distribute the URL identifying the publication in the public portal.

If the publication is distributed under the terms of Article 25fa of the Dutch Copyright Act, indicated by the "Taverne" license above, please follow below link for the End User Agreement:

www.tue.nl/taverne

Take down policy

If you believe that this document breaches copyright please contact us at:

openaccess@tue.nl

providing details and we will investigate your claim. 


\title{
Closed-Form Expression for BER of CE-OFDM in Optical Intensity-Modulated Direct-Detection Systems
}

\author{
Vinícius Oliari, Caio Santos, Pablo Marciano, Helder Rocha ${ }^{\circledR}$, Marcelo Segatto ${ }^{\circledR}$, and Jair Silva ${ }^{\circledR}$
}

\begin{abstract}
A closed-form expression for the bit-error-rate (BER) of constant envelope orthogonal frequency division multiplexing (CE-OFDM) in intensity-modulated direct-detection optical systems is provided in this letter. To the best of our knowledge, this letter is the first that provides an expression of the BER that considers the impact of electrical phase modulation index, signal bandwidth, optical modulation index, and Mach-Zehnder modulator bias point. Simulation and experimental results are included to validate the accuracy of the expression, subject to an analysis of the impact of electrical phase modulation index and the level of subcarrier mapping. With the analytical expression, we are able to reduce the computation time in design and parameter optimization of optical systems based in the CE-OFDM.
\end{abstract}

Index Terms-Constant envelope optical OFDM, bit error rate (BER), optical signal-to-noise ratio (OSNR).

\section{INTRODUCTION}

A FTER a brief experimental proof-of-concept provided in [1], the potential of the constant envelope orthogonal frequency division multiplexing (CE-OFDM) technique in intensity-modulated direct-detection (IMDD) optical systems is sustained mainly due to good tolerance to Mach-Zehnder modulator (MZM) intermodulation effects. This tolerance is especially evaluated for large values of the optical modulation index (OMI) that typically introduce extra nonlinearity terms from the MZM transfer function [2]. Indeed, the results discussed in [3] and [4] show that the CE-OFDM based optical systems outperform conventional OFDM systems regarding tolerance to uncompensated standard single-mode fiber (SSMF) nonlinearities such as Kerr effect, despite the spectral efficiency reduction that characterizes phase modulation schemes [5]. Initially proposed by S. C. Thompson et al. in [6], this peak-to-average power ratio (PAPR) reduction technique based on electrical phase modulation, was also adapted (PAPR $=3 \mathrm{~dB}$ ) for application in low-cost data-center interconnects using multimode fibers and $850-\mathrm{nm}$ verticalcavity surface-emitting lasers [7].

Consistently, all the above-cited works demonstrated the relevance of the phase modulation index $h$ in a tradeoff imposed between system performance and spectral broadening. The

Manuscript received April 30, 2019; revised June 13, 2019; accepted July 12, 2019. Date of publication July 17, 2019; date of current version October 9, 2019. The associate editor coordinating the review of this letter and approving it for publication was G. Contestabile. (Corresponding author: Jair Silva.)

V. Oliari is with the ICTLab, Department of Electrical Engineering, Eindhoven University of Technology, Eindhoven, The Netherlands.

C. Santos, P. Marciano, H. Rocha, M. Segatto, and Jair Silva are with the LabTel-DEL, University of Espírito Santo, Vitoria, Brazil (e-mail: jair.silva@ufes.br).

Digital Object Identifier 10.1109/LCOMM.2019.2929515 establishment of optimal performance regions (values of $h$ ) and ranges in which the system outcome is degraded by noise and modulation nonlinearity, becomes rhetoric in such CE-OFDM based systems. Among other parameters like signal bandwidth $\left(B_{e}\right)$ and MZM bias point $\left(V_{\text {bias }}\right)$, the optical modulation index presents itself as one of the most critical parameters in performance improvements of such optical IMDD systems [2], [8]. Manipulations in the CE-OFDM signal amplitude $(A)$ undoubtedly plays a crucial role in an expected performance and nonlinearity tradeoff [3], [4].

The necessity of a theoretical expression for the performance of the above mentioned system is raised at this point, since it provides insights into the performance dependence on the physical parameters of CE-OFDM in optical systems. Beyond the particular interest in the complex optimization of such optical systems, an analytical expression may allow obtaining accurate results in performance evaluations, with substantially reduced computation time [9]. Therefore, an exact closed-form expression for the bit error rate (BER) of optical IMDD systems based on CE-OFDM signals is provided in this letter, considering the impact of the above mentioned parameters $h, A, B_{e}$ and $V_{\text {bias }}$. Simulation and experimental results, generated exclusively to validate the derived analytic expression, show the accuracy of the BER evaluated in terms of optical signal-to-noise ratio (OSNR).

\section{THEORETICAL MODEL}

CE-OFDM is a modulation technique in which, a bandpass OFDM signal $x(t)=C \sum_{k=0}^{N_{s}} \Re[X(k)] \cos \left(\frac{2 \pi k t}{T}\right)-$ $\Im[X(k)] \sin \left(\frac{2 \pi k t}{T_{T}}\right)$; for $C$ a constant, $\{X(k)\}_{k=0}^{N_{s}}$ the data symbols, $T=\frac{N}{F_{s}}$ the signaling interval duration, $F_{s}$ the sampling rate and $N=2 N_{s}+2$ the fast-Fourier transform length; phase modulates an electrical carrier to decrease its inherit high PAPR [4]-[6]. Thereby, an electrical CE-OFDM signal, generated from $x(t)$ with mean power $\sigma_{s}^{2}$ and bandwidth $B_{W}$, can be expressed as

$$
c(t)=A \cos \left[2 \pi f_{c} t+\theta_{n}+2 \pi h \cdot x(t)\right],
$$

where $A$ is the signal amplitude, $f_{c}$ is the carrier frequency, $\theta_{n}$ is a phase continuous memory term and $h$ is the electrical phase modulation index. The bandwidth of $c(t)$ is usefully expressed as $B=\max (2 \pi h, 1) B_{e} \mathrm{~Hz}$, which is a rootmean-square bandwidth, lower bounded by $B_{e}$ [3]. Using this constant-envelope signal at its RF input, the optical field at the output of a single arm MZM, employed to modulate a continuous wave $(\mathrm{CW})$ laser source, can be written as

$$
E_{M Z M}(t)=\cos \left[\frac{\pi c(t)}{2 V_{\pi}}-\frac{\pi V_{\text {bias }}}{2 V_{\pi}}\right] \cdot \sqrt{2 P} \cos \left(w_{0} t\right),
$$


where $V_{\pi}$ and $V_{\text {bias }}$ are its switching and polarization voltages, $P$ is the laser power and $w_{0}=2 \pi f_{0}$ the angular frequency of the optical signal [10].

After pre-amplification and photodetection, the current $i_{P I N}$ at the output of a photodiode with responsivity $R$ is proportional to $R E_{P I N}^{2}$, with

$$
\begin{aligned}
E_{P I N}^{2} & (t) \\
= & \cos ^{2}\left[\frac{\pi}{2 V_{\pi}}\left(c(t)-V_{\text {bias }}\right)\right] \cdot 2 G P \cos ^{2}\left(2 \pi f_{0} t\right) \\
& +n_{i}^{2} \cos ^{2}\left(2 \pi f_{0} t\right)+n_{q}^{2} \sin ^{2}\left(2 \pi f_{0} t\right) \\
& +2 \cos ^{2}\left(2 \pi f_{0} t\right) n_{i}(t) \sqrt{2 G P} \cos \left[\frac{\pi}{2 V_{\pi}}\left(c(t)-V_{\text {bias }}\right)\right] \\
& +2 n_{q}(t) \sin \left(2 \pi f_{0} t\right) \cos \left(2 \pi f_{0} t\right) \\
& \times\left\{n_{i}(t)+\sqrt{2 G P} \cos \left[\frac{\pi}{2 V_{\pi}}\left(c(t)-V_{\text {bias }}\right)\right]\right\},
\end{aligned}
$$

for $G$ the amplification gain, $n_{i}(t)$ and $n_{q}(t)$ the in-phase and quadrature noise components with power spectral density given by $N_{A S E} / 2$ and variance $\sigma_{n}^{2}=N_{A S E} \cdot B_{0}$, for $B_{0}$ the bandwidth of the optical filter (OF) at its output.

After some simplifications with trigonometric identities and neglecting high frequency contributions $\left(f>f_{0}+B_{0} / 2\right)$,

$$
\begin{aligned}
i_{P I N}(t)= & \frac{R}{2}\left\{G P\left[1+\cos \left(\frac{\pi}{V_{\pi}}\left(c(t)-V_{\text {bias }}\right)\right)\right]\right. \\
& +2 n_{i}(t) \sqrt{2 G P} \cos \left(\frac{\pi}{2 V_{\pi}}\left(c(t)-V_{\text {bias }}\right)\right) \\
& \left.+n_{i}^{2}(t)+n_{q}^{2}(t)\right\} .
\end{aligned}
$$

Neglecting the signal contributions at $f \geq 2 f_{c}$ due to a filtering process, a first-order Taylor expansion gives an approximation error in the SNR calculation. This error, depending on the system parameters, can be around $10 \%$ with respect to the eleventh-order, which affects the BER curves in a negligible manner. Thus, with the first-order Taylor expansion enabling $\cos \left(a_{1} c(t)+a_{2}\right) \approx \cos \left(a_{2}\right)-a_{1} c(t) \sin \left(a_{2}\right)$, for $a_{1}, a_{2} \in \mathbb{R}$, the received signal becomes

$$
\begin{aligned}
i_{P I N}(t) \approx & \frac{R}{2}\left\{G P\left[1+\cos \left(\frac{\pi V_{\text {bias }}}{V_{\pi}}\right)+\frac{\pi c(t)}{V_{\pi}} \sin \left(\frac{\pi V_{\text {bias }}}{V_{\pi}}\right)\right]\right. \\
& +2 n_{i}(t) \sqrt{2 G P}\left[\cos \left(\frac{\pi V_{\text {bias }}}{2 V_{\pi}}\right)+\frac{\pi c(t)}{2 V_{\pi}} \sin \left(\frac{\pi V_{\text {bias }}}{2 V_{\pi}}\right)\right] \\
& \left.+n_{i}^{2}(t)+n_{q}^{2}(t)\right\} .
\end{aligned}
$$

If $n_{i}^{2}(t)$ and $n_{q}^{2}(t)$ are significantly smaller than the optical carrier, after filtering the DC-component, the photocurrent can be simplified to

$$
i_{P I N}(t) \approx K_{1} \cos \left[2 \pi f_{c} t+2 \pi h x(t)\right]+K_{2}(t) n_{i}(t),
$$

$$
\begin{aligned}
& \text { for } \\
& \qquad \begin{aligned}
K_{1} & =\frac{A R G P \pi \sin \left(\frac{\pi V_{\text {bias }}}{V_{\pi}}\right)}{2 V_{\pi}}, \\
K_{2}(t) & =R \sqrt{2 G P}\left[\cos \left(\frac{\pi V_{\text {bias }}}{2 V_{\pi}}\right)+\frac{\pi c(t)}{2 V_{\pi}} \sin \left(\frac{\pi V_{\text {bias }}}{2 V_{\pi}}\right)\right] .
\end{aligned}
\end{aligned}
$$

Neglecting high frequency contributions and applying the first-order Taylor expansion after downconversion by a multiplication with $\cos \left(2 \pi f_{c} t+\frac{\pi}{2}\right)$, the signal $\sqrt{S}$ and noise $\sqrt{N}$ components (by an abuse of notation) can be written as

$$
\sqrt{S} \approx \frac{R}{2} G P\left[\frac{\pi A}{2 V_{\pi}} 2 \pi h x(t) \sin \left(\frac{\pi V_{\text {bias }}}{V_{\pi}}\right)\right]
$$

and

$$
\begin{aligned}
\sqrt{N} \approx \frac{R}{2}\{2 \sqrt{2 G P} & {\left[\cos \left(\frac{\pi V_{\text {bias }}}{2 V_{\pi}}\right) \cos \left(2 \pi f_{c} t+\frac{\pi}{2}\right)\right.} \\
+ & \left.\left.\frac{\pi A}{4 V_{\pi}} \sin \left(\frac{\pi V_{\text {bias }}}{2 V_{\pi}}\right) 2 \pi h x(t)\right]\right\} n_{i}(t),
\end{aligned}
$$

respectively. Hence, the SNR can be expressed as (11), shown at the bottom of this page.

Within a reference bandwidth of $B_{0}^{\prime}=12.5 \mathrm{GHz}(0.1 \mathrm{~nm})$, the optical SNR can be defined as OSNR $=P_{\text {med }} B_{0} /$ $\left(2 \sigma_{n}^{2} B_{0}^{\prime}\right)$ and consequently $\sigma_{n}^{2}=P_{\text {med }} B_{0} /\left(2 \mathrm{OSNR} B_{0}^{\prime}\right)$ [11]. Assuming that the optical power of the signal is determined mainly by the optical carrier [8] and the two electrical carriers (see Fig. 1.(b)), setting $x(t)=0$ in (3) is a reasonable approximation for calculating the optical mean power $P_{\text {med }}=$ $G P I_{\text {med }}$, for $I_{\text {med }}=\frac{1}{T} \int_{0}^{T} \cos ^{2}\left[\frac{\pi}{2 V_{\pi}}\left(A \cos \left(2 \pi f_{c} t\right)-V_{\text {bias }}\right)\right] \mathrm{d} t$ over the period $T=1 / f_{c}$. Then

$$
\begin{aligned}
& \left(\frac{S}{N}\right) \\
& =\left(\frac{B_{0}^{\prime}}{B_{0}}\right) \times\left(\frac{\text { OSNR }}{I_{\text {med }}}\right) \\
& \quad \times \frac{(2 \pi h)^{2} \sigma_{s}^{2} \pi^{2} A^{2} \sin ^{2}\left(\frac{\pi V_{b i a s}}{V_{\pi}}\right)}{\left[8 V_{\pi}^{2} \cos ^{2}\left(\frac{\pi V_{\text {bias }}}{2 V_{\pi}}\right)+\pi^{2} A^{2}(2 \pi h)^{2} \sigma_{s}^{2} \sin ^{2}\left(\frac{\pi V_{\text {bias }}}{2 V_{\pi}}\right)\right]}
\end{aligned}
$$

Therefore, considering that the error probability for quadrature phase-shift keying (QPSK, $M=4$ ) modulated data with additive Gaussian noise is $P_{b}=\frac{1}{2} \operatorname{erfc}\left(\sqrt{\frac{\mathrm{SNR}}{\log _{2}(M)}}\right)$ [12], the system bit-error ratio (BER) is given by equation (13), as shown at the bottom of this page, where $B_{0} / 2$ is replaced by the single-sided electrical bandwidth $B_{e}$.

$$
\left(\frac{S}{N}\right)=\frac{(2 \pi h)^{2} \sigma_{s}^{2} \pi^{2} A^{2} \sin ^{2}\left(\frac{\pi V_{b i a s}}{V_{\pi}}\right) G P}{\sigma_{n}^{2}\left[16 V_{\pi}^{2} \cos ^{2}\left(\frac{\pi V_{b i a s}}{2 V_{\pi}}\right)+2 \pi^{2} A^{2}(2 \pi h)^{2} \sigma_{s}^{2} \sin ^{2}\left(\frac{\pi V_{b i a s}}{2 V_{\pi}}\right)\right]} .
$$

$$
\mathrm{BER}=\frac{1}{2} \operatorname{erfc}\left(\sqrt{\frac{1}{2}\left(\frac{B_{0}^{\prime}}{2 B_{e}}\right)\left(\frac{2 \cdot \mathrm{OSNR}}{1+J_{0}\left(\frac{\pi A}{V_{\pi}}\right) \cos \left(\frac{\pi V_{\text {bias }}}{V_{\pi}}\right)}\right) \frac{(2 \pi h)^{2} \sigma_{s}^{2} \pi^{2} A^{2} \sin ^{2}\left(\frac{\pi V_{\text {bias }}}{V_{\pi}}\right)}{\left[8 V_{\pi}^{2} \cos ^{2}\left(\frac{\pi V_{\text {bias }}}{2 V_{\pi}}\right)+\pi^{2} A^{2}(2 \pi h)^{2} \sigma_{s}^{2} \sin ^{2}\left(\frac{\pi V_{\text {bias }}}{2 V_{\pi}}\right)\right]}}\right)
$$


(a) Experimental setup
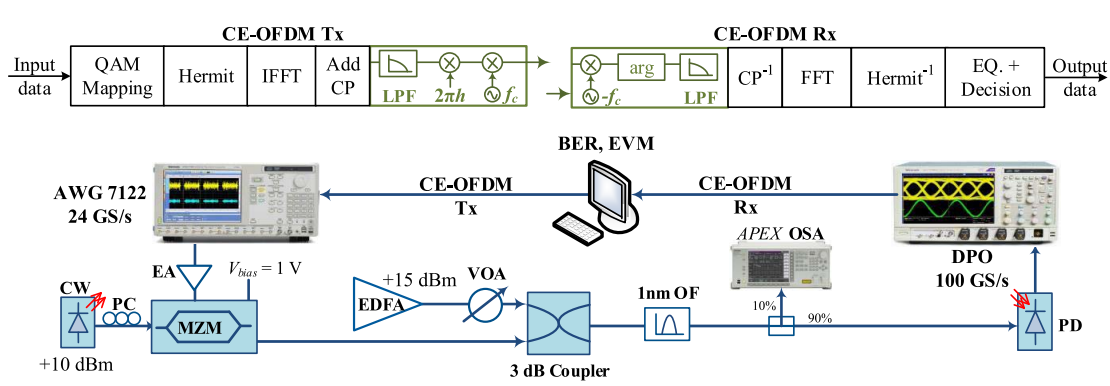

(b) Measured optical spectrum

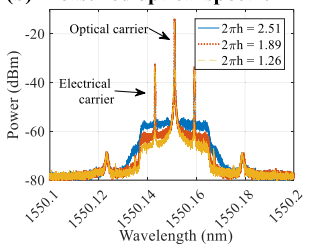

(c) OSNR measuremen

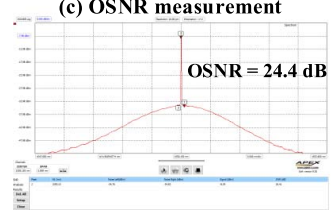

Fig. 1. (a) Experimental setup of the DDO-CE-OFDM system. EA: electrical amplification; PC: polarization controller; VOA: variable optical attenuator; Sync: synchronism. (b) Spectrum of the optical CE-OFDM signals. (c) An illustration of the OSNR estimation method.

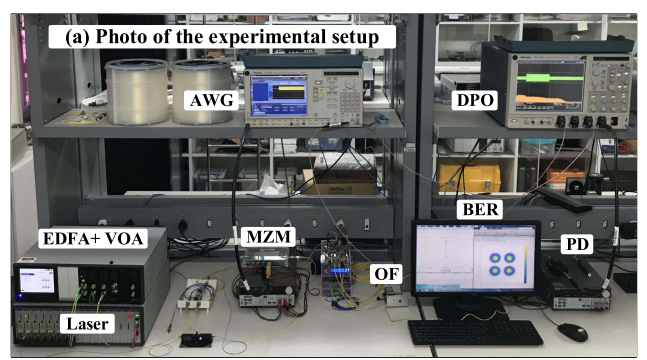

Fig. 2. A picture of the prepared experimental setup.

$I_{m e d}$ is replaced by the demonstration performed in the Appendix, in which $J_{0}(\cdot)$ is the $0 t h$-order modified Bessel function of the first kind. For quadrature amplitude modulation (QAM), with $M>4$, the BER expression can be derived from $P_{b}=\frac{2}{\log _{2}(M)} \operatorname{erfc}\left(\sqrt{\frac{3 \mathrm{SNR}}{2(M-1)}}\right)$, which is the upper bound (4.3-30) described in Ref. [12].

\section{VALidATION OF THE BER ClOSED-Form}

\section{A. Experimental Setup}

Block diagrams of the setup used to validate the derived BER expression are illustrated in Fig. 1. Pseudorandom binary sequences PRBS $=\log _{2}(M) \times\left(2^{9}\right)$ were mapped in QPSK and 16-QAM $(M=16)$ before Hermitian symmetry processing and multiplexing of 255 data subcarriers using an inverse fast-Fourier transform (IFFT) size of 512. The ordinary cyclic prefix (CP) is added before windowing and low pass filtering (LPF). Then, the OFDM signals with $\sigma_{s} \approx 0.2$ and bandwidth $B_{e}=3.0 \mathrm{GHz}$ modulates the phase of a carrier centered at $f_{c}=4.5 \mathrm{GHz}$, after signal multiplication with the phase modulation index $2 \pi h$ [3], [4].

The 300 CE-OFDM signals with PAPR $=3 \mathrm{~dB}$, performed offline using MATLAB, are loaded to the 24 Gsamples/s arbitrary waveform generator (AWG7122), before analog lowpass filtering by a $25 \mathrm{GHz}$ amplifier. Employing a conventional single-arm MZM biased at $V_{\text {bias }}=0.576 \times V_{\pi}$ for $V_{\pi}=6.6 \mathrm{~V}$, the bandpass signals modulate the laser source centered at $\lambda=1550 \mathrm{~nm}$. The picture showed in Fig. 2 portrays details about the prepared experimental apparatus.

A photodiode is used to detect the optical double-sideband signals shown in Fig. 1.(b), measured at the output of the OF. After a sampling process by the 100 Gsamples/s oscilloscope, the recorded photocurrents were processed offline. A discrete phase demodulation, provided by an arctangent (arg) signal processing and a phase unwrapping, is implemented before conventional demultiplexing of the OFDM signals impaired by linear channel distortions [3], [4]. The arg block extracts the phase of the baseband signals through the argument obtained from the demodulated input samples [6].

In order to analyze the effect of optical noise in the system performance, white noise, generated by an Erbium-doped fiber amplifier (EDFA) followed by a variable optical attenuator (VOA), is added by a $3 \mathrm{~dB}$ coupler. A $1 \mathrm{~nm}$ optical bandpass filter (OF) is placed after the coupler to limit the bandwidth of the optical noise. For the OSNR measurements, we used the interpolation method described in Section 9.5.1 of Ref. [13]. This method consists of measuring the signal power at the desired frequency and measuring the noise power at points determined by the interpolation offset. The noise measurements are used to interpolate the noise power at the signal frequency and the OSNR is then estimated by subtracting the signal power from the interpolated noise power. Thereon, we used an APEX OSA with a reference optical bandwidth of $0.1 \mathrm{~nm}$ and an inserted offset equal to $50 \mathrm{pm}$, as seen in Fig. 1.(c).

\section{B. Monte Carlo Simulations}

In order to compare the results of the analytical expression with the results obtained by a numerical model, we conduct Monte Carlo simulations of the system depicted in Fig. 3 of Ref. [3], in optical back-to-back, without the single-sideband optical filter and considering only the effect of the amplified spontaneous emission (ASE) noise. We assume that the ASE is the dominant noise ${ }^{1}$ source and it is modeled as an optical additive white Gaussian noise (AWGN).

The CE-OFDM signals were generated with the same parameters described in Section III-A and, to assure a fair performance comparison, we adapt the parameters $\left(V_{\pi}=6.6 \mathrm{~V}, V_{\text {bias }}=3.8 \mathrm{~V}\right)$ of the conventional MZM model characterized in the work outlined in [9].

\section{Results and Discussions}

Figure 3 depicts a performance comparison between the derived closed-form, the experimental and the numerical

\footnotetext{
${ }^{1}$ This is a reasonable assumption when the erbium-doped fiber amplifiers of long-haul systems have high gains.
} 


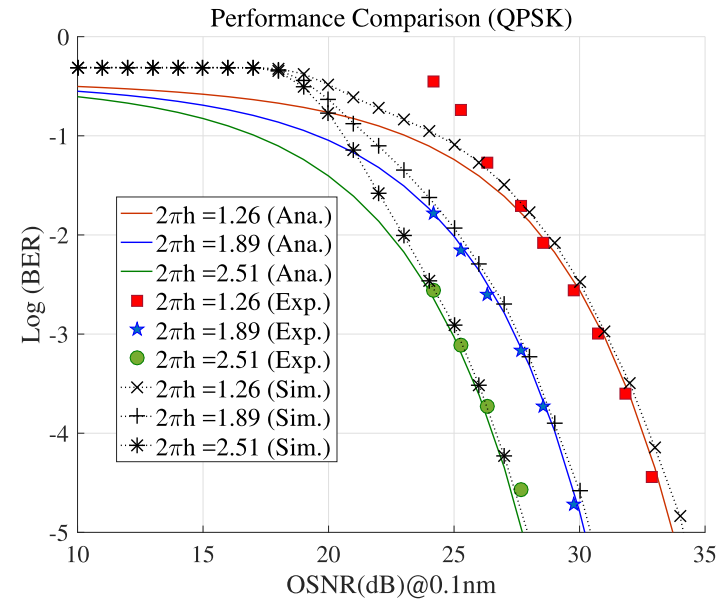

Fig. 3. BER versus OSNR for different values of $2 \pi h$, for QPSK.

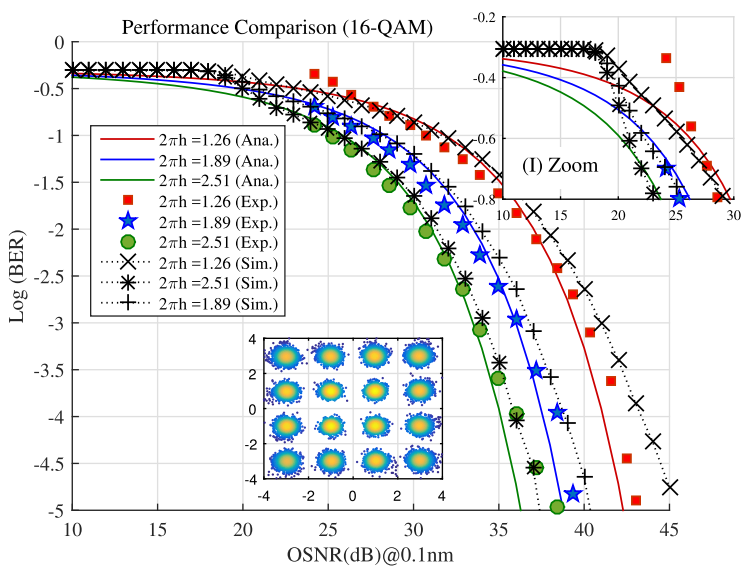

Fig. 4. BER versus OSNR for 16-QAM. The constellation shown inset was measured for $2 \pi h=1.89$ and OSNR $=39 \mathrm{~dB}$.

results obtained to substantiate the accuracy of the BER expression, for QPSK mapping.

As expected, Fig. 3 clearly show that the system performance strongly depends on the electrical phase modulation index [3], [4]. The agreement between the theoretical, the experimental measurements and the simulation results depicted in Fig. 3 demonstrates the accuracy of the derived BER expression, for all considered indexes.

In the case of 16-QAM, the agreement depends on the OSNR and the employed phase index, as depicted in Fig. 4. In particular, Fig. 4 indicates that, as suggested by the discussions presented in [3] and [4], this agreement is almost achieved for the values of $2 \pi h$ in the linear regions. An OSNR difference of $\approx 1 \mathrm{~dB}$ is registered for $2 \pi h=2.51$ at a $\mathrm{BER}=10^{-4}$, when the theoretical performance is compared with the experimental and the simulation results. This difference increases to $\approx 1.2 \mathrm{~dB}$ for $2 \pi h=1.89$, whereas for $2 \pi h=1.26$ it is almost $2 \mathrm{~dB}$ when compared to the result obtained by the numerical model.

It is straightforwardly noticed from Fig. 4. that, for OSNR $\geq 40 \mathrm{~dB}$ at $2 \pi h=1.26$, the expression doesn't agree with the experimental and especially with the numerical results. This is explained by the phase noise limitations that inflicts such high level subcarrier mappings, at relatively low values of $2 \pi h$ [6], [14]. The small differences occurred in the performances, particularly evidenced for the large values of phase index $(2 \pi h=2.51)$ at OSNRs greater than $36 \mathrm{~dB}$, are due to the nonlinear nature of the evaluated phase modulation. It should be stressed that this behavior is not contemplated by the BER closed-form. A well designed finite impulse response filter with Hamming window, preceding the phase demodulation, can be used to improve the performance of the numerical model [14].

Figs 3 and 4 also show that, at low values of SNR (the low carrier-to-noise ratios depicted in Fig. 2 of [6]), the system performances degrade drastically. In this case, the closed-form is invalid and both systems are said to be below a threshold (see Fig. 3 of [6]), above which the noise is modeled as a sample function of a zero mean Gaussian process.

\section{CONCLUSiON}

A closed-form expression for the bit error rate of constant envelope OFDM in intensity-modulated direct-detection optical systems was provided. Numerical and experimental results were obtained to validate the accuracy of the derived expression, demonstrating excellent agreement with the analytical theory, depending on the phase modulation index.

Beyond the phase index, we provided performance comparisons for different subcarrier modulation levels. The results also show the intended accordance between the theoreti$\mathrm{cal}$, the numerical and the experimental performance curves, depending on the aforementioned modulation index and the optical signal-to-noise ratio.

Henceforth, with the computation time reduction provided by the BER expression, we are able to quickly design and optimize optical IMDD with constant envelope signals.

$$
\begin{aligned}
I_{\text {med }} & =\frac{1}{T} \int_{0}^{T} \cos ^{2}\left(\frac{\pi A \cos \left(2 \pi f_{c} t\right)-\pi V_{\text {bias }}}{2 V_{\pi}}\right) \mathrm{d} t \\
& =\frac{1}{2}+\frac{\cos \left(\frac{\pi V_{\text {bias }}}{V_{\pi}}\right)}{2 \pi} \int_{0}^{\pi} \cos \left(\frac{\pi A \sin (\theta)}{V_{\pi}}\right) \mathrm{d} \theta \\
& =\frac{1}{2}+\frac{J_{0}\left(\frac{\pi A}{V_{\pi}}\right) \cos \left(\frac{\pi V_{\text {bias }}}{V_{\pi}}\right)}{2} .
\end{aligned}
$$

\section{ACKNOWLEDGEMENTS}

The authors acknowledge the support from the FAPES 80599230/17, 538/2018, 84343338, 601/2018, and CNPq $307757 / 2016-1,304564 / 2016-8,309823 / 2018-8$ projects. The authors would like to thank Gabriele Liga.

\section{REFERENCES}

[1] J. A. L. Silva, T. Alves, A. Cartaxo, and M. E. V. Segatto, "Experimental demonstration of a direct-detection constant envelope OFDM system," in Proc. Adv. Photon. Renew. Energy, 2010, pp. 1-3, Paper SPThB2.

[2] W. R. Peng et al., "Theoretical and experimental investigations of directdetected RF-tone-assisted optical OFDM systems," J. Lightw. Technol., vol. 27, no. 10, pp. 1332-1339, May 15, 2009.

[3] J. A. L. Silva, A. V. T. Cartaxo, and M. E. V. Segatto, "A PAPR reduction technique based on a constant envelope OFDM approach for fiber nonlinearity mitigation in optical direct-detection systems," IEEE/OSA J. Opt. Commun. Netw., vol. 4, no. 4, pp. 296-303, Apr. 2012. 
[4] R. B. Nunes, H. R. de O. Rocha, M. E. V. Segatto, and J. A. L. Silva, "Experimental validation of a constant-envelope OFDM system for optical direct-detection," Opt. Fiber Technol., vol. 20, no. 3, pp. 303-307, 2014.

[5] C.-D. Chung, "Spectral precoding for constant-envelope OFDM," IEEE Trans. Commun., vol. 58, no. 2, pp. 555-567, Feb. 2010.

[6] S. C. Thompson, A. U. Ahmed, J. G. Proakis, J. R. Zeidler, and M. J. Geile, "Constant envelope OFDM," IEEE Trans. Commun., vol. 56, no. 8, pp. 1300-1312, Aug. 2008.

[7] R. B. Nunes, H. R. de O. Rocha, D. A. A. Mello, F. D. Simões, M. E. V. Segatto, and J. A. L. Silva, "Transmission of CE-OFDM signals over MMF links using directly modulated 850-nm VCSELs," IEEE Photon. Technol. Lett., vol. 27, no. 3, pp. 315-318, Feb. 1, 2015.

[8] J. Leibrich, A. Ali, H. Paul, W. Rosenkranz, and K.-D. Kammeyer, "Impact of modulator bias on the OSNR requirement of directdetection optical OFDM," IEEE Photon. Technol. Lett., vol. 21, no. 15, pp. 1033-1035, Aug. 1, 2009.
[9] E. da V. Pereira, H. R. de O. Rocha, R. B. Nunes, M. E. V. Segatto, and J. A. L. Silva, "Impact of optical power in the guard-band reduction of an optimized DDO-OFDM system," J. Lightw. Technol., vol. 33, no. 23, pp. 4717-4725, Dec. 1, 2015.

[10] D. J. F. Barros and J. M. Kahn, "Optimized dispersion compensation using orthogonal frequency-division multiplexing," J. Lightw. Technol., vol. 26, no. 16, pp. 2889-2898, Aug. 15, 2008.

[11] I. Kaminow, T. Li, and A. E. Willner, Optical Fiber Telecommunications $V B$ : Systems and Networks, 5th ed. Amsterdam, The Netherlands: Elsevier, 2008.

[12] J. Proakis and M. Salehi, Digital Communications, 5th ed. Boston, MA, USA: McGraw-Hill, 2007.

[13] Series G: Transmission Systems and Media Digital Systems and Networks, ITU-T, Geneva, Switzerland, 2012.

[14] S. C. Thompson, J. G. Proakis, and J. R. Zeidler, "Noncoherent reception of constant envelope OFDM in flat fading channels," in Proc. IEEE 16th Int. Symp. Pers., Indoor Mobile Radio Commun., vol. 1, Sep. 2005 , pp. 517-521. 